\title{
Circulating Tumor Cells in metastatic lung cancer enriched by EpCAM expression and physical characteristics
}

Sanne de Wit ${ }^{\star}$, Guus van Dalum ${ }^{1 \#}$, Joost van Dalum ${ }^{1}$, Aufried T.M. Lenferink ${ }^{1}$, Arjan G.J. Tibbe ${ }^{2}$, Cees J.M. van Rijn ${ }^{3}$, T. Jeroen N. Hiltermann ${ }^{4}$, Harry J.M. Groen ${ }^{4}$, Leon W.M.M. Terstappen ${ }^{1}$ Department of Medical Cell BioPhysics, MIRA institute, University of Twente, Enschede, The Netherlands; ${ }^{2}$ VyCAP, Deventer, The Netherlands;
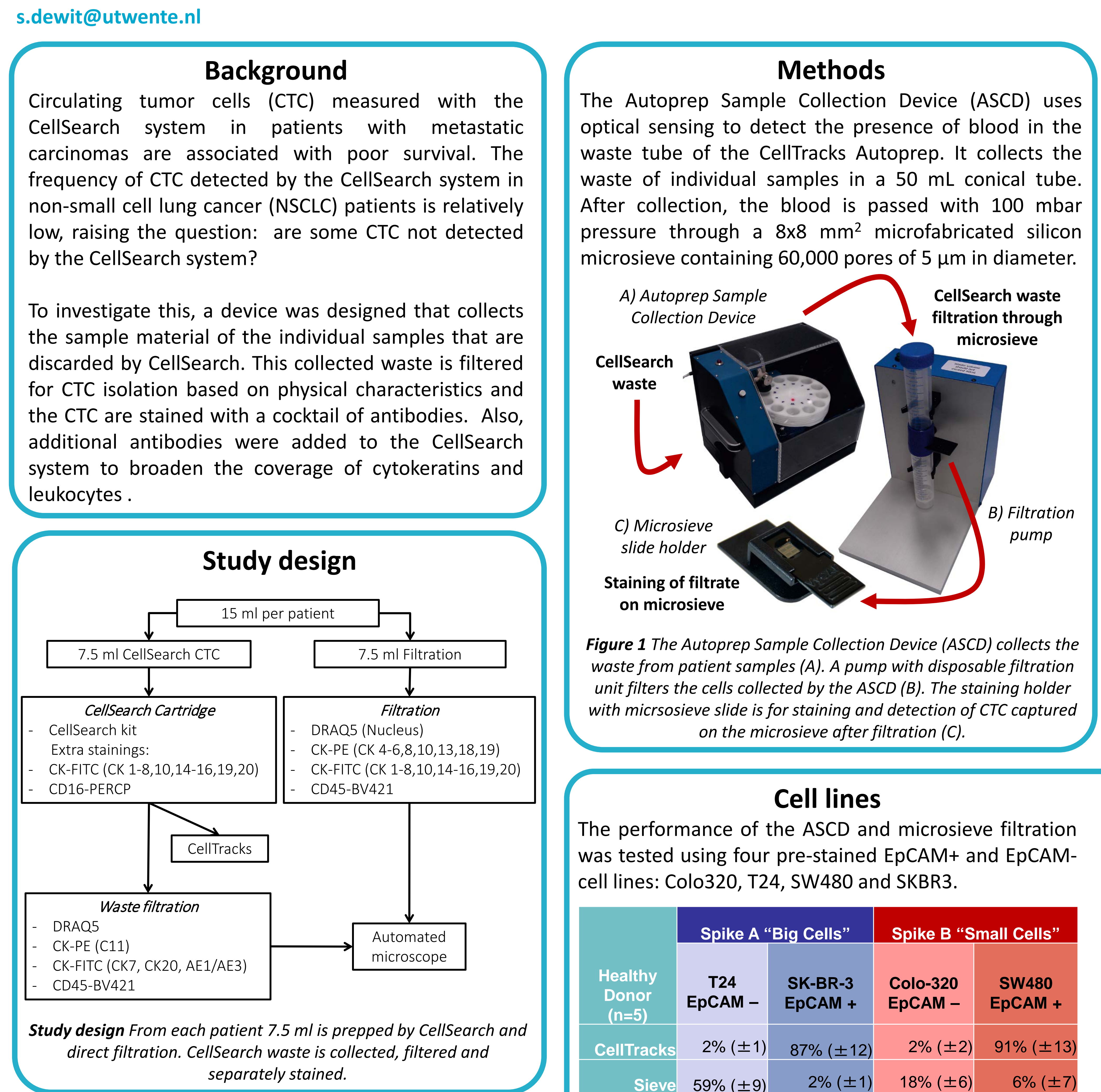

Cell lines
The performance of the ASCD and microsieve filtration cell lines: Colo320, T24, SW480 and SKBR3.

$$
\text { s. }
$$

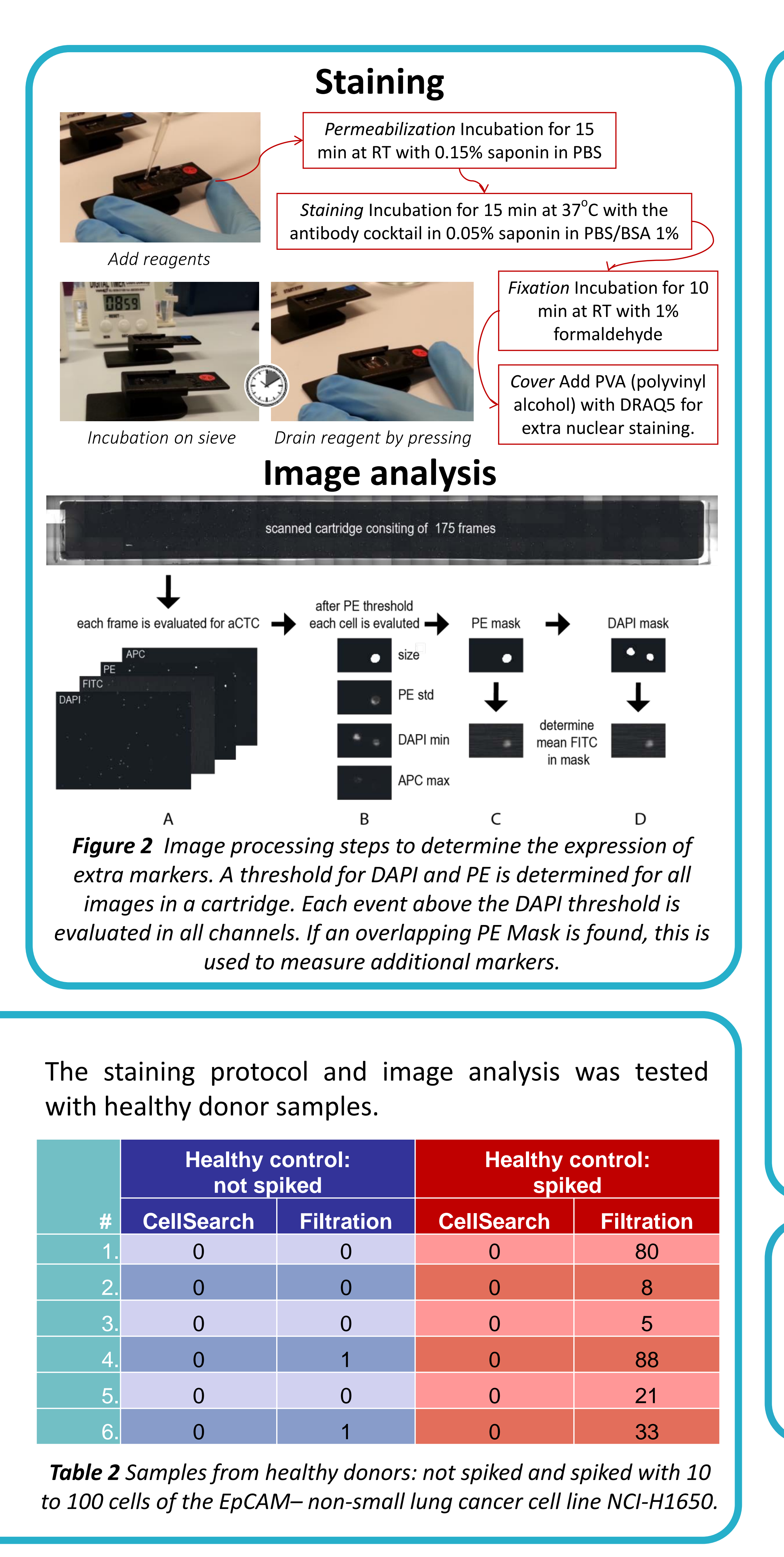

Preliminary results
Patients with NCSLC (enrollment is ongoing) were processed on the

Patients with NCSLC (enrollment is ongoing) were processed on the Cellsearch and stained with a nucleic acid dye and antibodies recognizing leukocytes and all cyieves were Additional antibodies were added to the Cellsearch test to cover all cytokeratins and broaden the coverage of leukocytes.

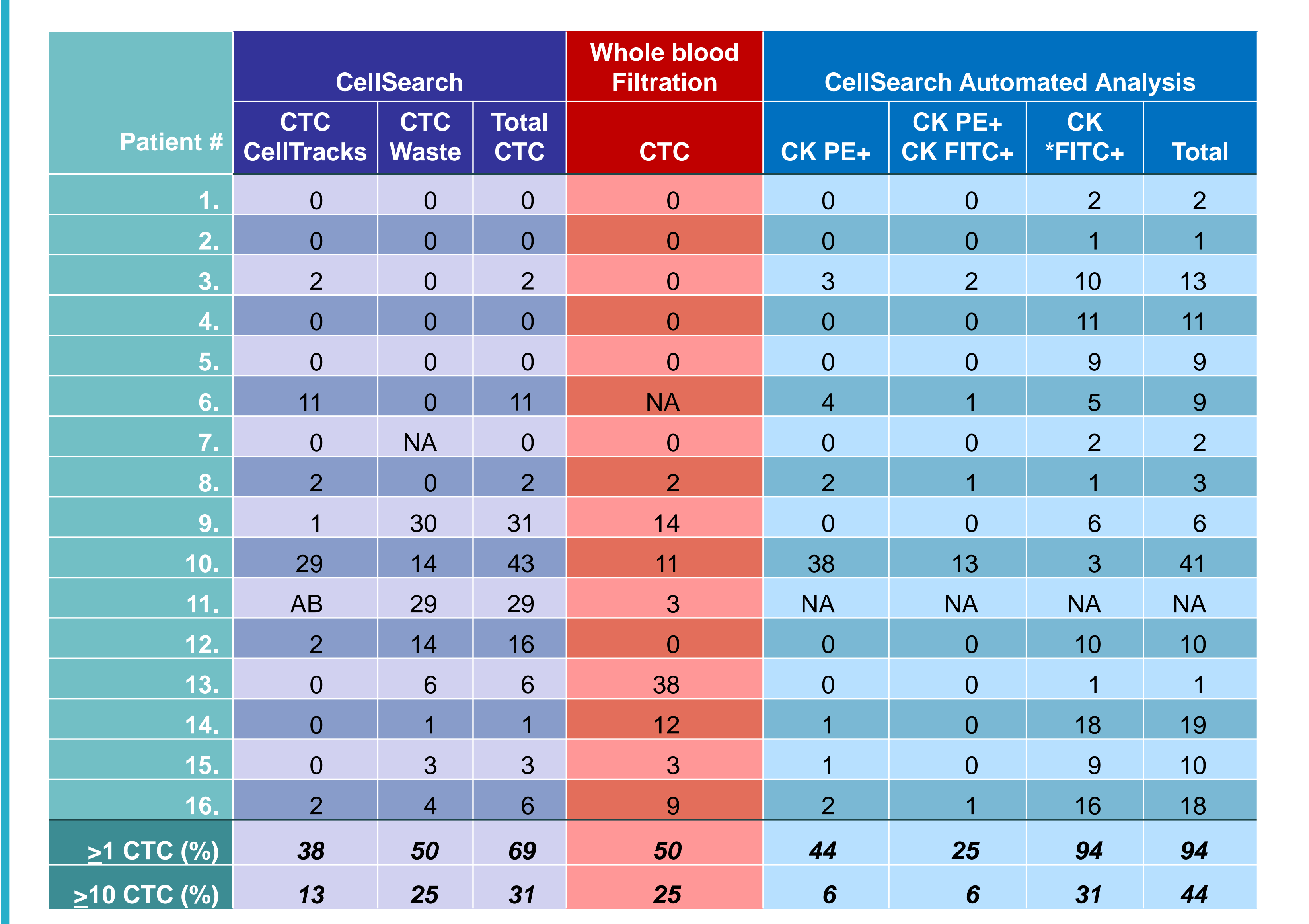
Table 3 Overview of CTC found in NSCLC patients, *Some CK-FITCC events are due to debris which would be
discarded in a manual count. demonstrated that low EpCAM expression results in the presence of CTC in the waste, that otherwise would not be detected by the CellSearch system. In NSCLC additional CTC can be detected but it still remains to be determined whether these CTC - not detected by the original CellSearch approach - are also of clinical relevance.

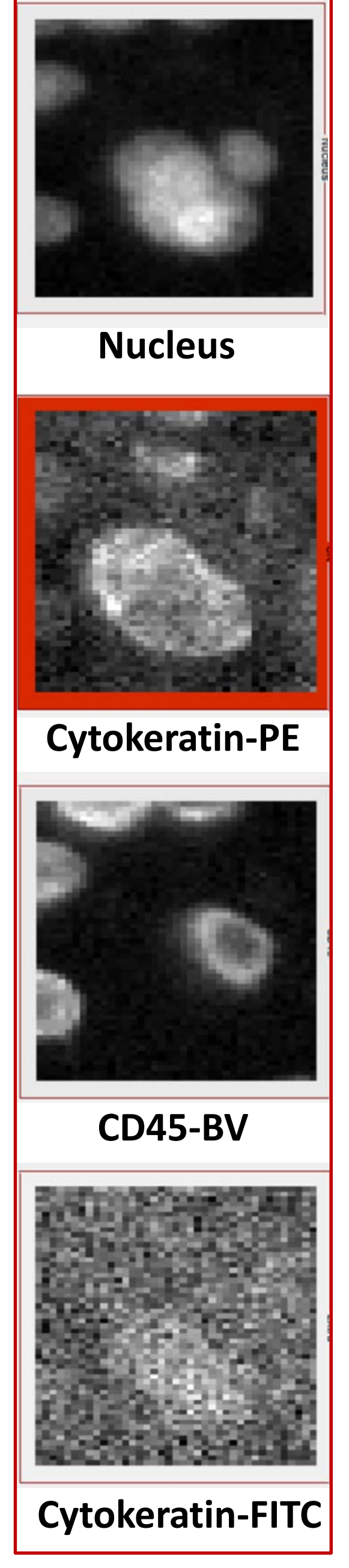

Gure 3 Thumbnail gallary

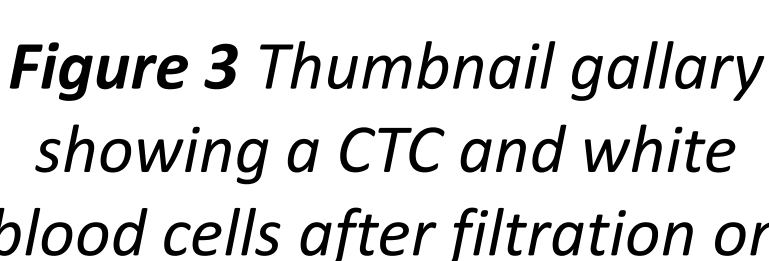

this
be
ned

